\title{
Short communication: Imputation of markers on the bovine $\mathrm{X}$ chromosome
}

\author{
Xiaowei Mao, ${ }^{*}{ }^{1}$ Anna Maria Johansson, $\dagger$ Goutam Sahana, ${ }^{*}$ Bernt Guldbrandtsen, ${ }^{*}$ and Dirk-Jan De Koning \\ *Department of Molecular Biology and Genetics, Center for Quantitative Genetics and Genomics, Aarhus University, PO Box 50, DK-8830, \\ Tjele, Denmark \\ †Department of Animal Breeding and Genetics, Swedish University of Agricultural Sciences, Box 7023, 75007 Uppsala, Sweden
}

\section{ABSTRACT}

Imputation is a cost-effective approach to augment marker data for genomic selection and genome-wide association studies. However, most imputation studies have focused on autosomes. Here, we assessed the imputation of markers on the $\mathrm{X}$ chromosome in Holstein cattle for nongenotyped animals and animals genotyped with low-density (Illumina BovineLD, Illumina Inc., San Diego, CA) chips, using animals genotyped with medium-density (Illumina BovineSNP50) chips. A total of 26,884 genotyped Holstein individuals genotyped with medium-density chips were used in this study. Imputation was carried out using FImpute V2.2. The following parameters were examined: treating the pseudoautosomal region as autosomal or as X specific, different sizes of reference groups, different male/female proportions in the reference group, and cumulated degree of relationship between the reference group and target group. The imputation accuracy of markers on the $\mathrm{X}$ chromosome was improved if the pseudoautosomal region was treated as autosomal. Increasing the proportion of females in the reference group improved the imputation accuracy for the $\mathrm{X}$ chromosome. Imputation for nongenotyped animals in general had lower accuracy compared with animals genotyped with the low-density single nucleotide polymorphism array. In addition, higher cumulative pedigree relationships between the reference group and the target animal led to higher imputation accuracy. In the future, better marker coverage of the $\mathrm{X}$ chromosome should be developed to facilitate genomic studies involving the $\mathrm{X}$ chromosome.

Key words: imputation, pseudoautosomal region, $\mathrm{X}$ chromosome, nongenotyped

Received March 11, 2016.

Accepted June 3, 2016.

${ }^{1}$ Corresponding author: xiaowei.mao@mbg.au.dk

\section{Short Communication}

Both genomic selection (Meuwissen et al., 2001) and genome-wide association studies (McCarthy et al., 2008) require genotyping of large numbers of individuals, which can be costly and time consuming. To cope with these problems, young cattle are frequently genotyped with a relatively cheap, lower density panel or not genotyped at all. Genotypes may be imputed to a higher density panel using information from a reference population (Marchini and Howie, 2010). The genotypes of nongenotyped animals may to some extent be inferred from their relatives' genotypes. The majority of imputation studies in cattle have examined the accuracy of imputation from low-density to high-density SNP arrays (Ma et al., 2013; Brøndum et al., 2014; Pryce et al., 2014), but only a few studies have imputed nongenotyped animals to a higher density (Boison et al., 2014; Bouwman et al., 2014; Sargolzaei et al., 2014).

However, most of these studies focused on imputation of autosomes (Brøndum et al., 2014; van Binsbergen et al., 2014). Hickey and Kranis (2013), using 1,255 individuals from a pedigree of 4 generations, showed that the $\mathrm{Z}$ chromosome in chickens with 25 SNP could be imputed to a higher density $(1,137$ SNP) with relatively high accuracy (approximately 0.9). However, this study did not consider the pseudoautosomal region (PAR) that recombines with the $\mathrm{W}$ chromosome. In dairy cattle, ignoring the $\mathrm{X}$ chromosomes could miss important biological functions and affect genomic evaluation (Sandor et al., 2006; Lyons et al., 2014; Su et al., 2014; de Camargo et al., 2015). Regarding biological functions, nonsynonymous mutations on the $\mathrm{X}$ chromosome were shown to be associated with andrological and growth traits in beef cattle (Lyons et al., 2014). The $\mathrm{X}$ chromosome is important due to its relatively long length $(148,823,899 \mathrm{bp})$, accounting for approximately $6 \%$ of the total physical genome (Zimin et al., 2009). ENSEMBL (release 82) lists 19,981 protein coding genes in the cattle genome out of which $833(4.2 \%)$ are found on the $\mathrm{X}$ chromosome. Regarding the genomic evaluation, Su et al. (2014) reported that incorporating markers on the $\mathrm{X}$ chromosome to genomic predictions 
contributes 0.3 to $0.5 \%$ to the reliability of genomic predictions. They also reported that the $\mathrm{X}$ chromosome accounted for on average $1.7 \%$ of the total additive genetic variance for 15 indices included in the Nordic Total Merit index (http://www.nordicebv.info). However, in their study only genotypes from males were used. Moreover, imputation of the $\mathrm{X}$ chromosome for nongenotyped animals has not been investigated in these studies. Imputing genotypes for nongenotyped animals with phenotypic records, especially for records that are difficult or expensive to measure, could potentially provide new insights.

The objective of our study was to assess the imputation accuracy of markers on the $\mathrm{X}$ chromosome in dairy cattle for nongenotyped animals and animals genotyped with low-density chips.

All data were recorded for routine dairy cattle management and genetic evaluations, and no additional tests or perturbations were performed for the purpose of this study. Our data set contained 26,884 animals. By breed, these 26,884 animals included 26,383 Holstein and 501 Red Holstein; by sex, they included 17,996 males and 8,888 females. These animals were genotyped by Illumina BovineSNP50 BeadChip version 1 and 2 (50K; Illumina Inc., San Diego, CA; Matukumalli et al., 2009). The minimal accepted GenCall score for individual genotype was 0.60 . There were a total of 55,298 SNP in the 50K chips including SNP on 29 BTA as well as the X chromosome. The number of SNP included for analysis varied between 2,502 on the BTA1 and 894 on the X chromosome. The UMD v3.1 assembly (Zimin et al., 2009) was used as the reference for genomic position.

The PAR is homologous between the mammalian $\mathrm{X}$ and $\mathrm{Y}$ chromosomes. It is required for sex chromosome segregation during male meiosis (Das et al., 2009). The boundary between the PAR and X-specific region was assigned to the $5^{\prime}$ end of the GPR143 gene (chromosome X: 143,861,798-143,891,357) in previous studies using sequence-based analysis and fluorescence in situ hybridization (Van Laere et al., 2008; Das et al., 2009). Thus the PAR in our study was defined from $143,861,798$ to $148,823,899 \mathrm{bp}$ on the X chromosome. Heterozygosity of the $\mathrm{X}$ chromosome was also calculated using genotypes of 17,996 males to confirm our definition of the PAR, because males should be completely homozygous for the non-PAR region (barring unusual conditions such as XXY genotypes). The rest of the SNP on the X chromosome were defined as X-linked SNP.

Imputation was carried out using FImpute V2.2 (Sargolzaei et al., 2014), which combines both family and population-based information. Different scenarios of imputation were set up to evaluate the effect of the following factors on imputation accuracy: treating SNP in the PAR either as autosomal or as X-linked, different sizes of reference groups, different proportions of males and females in the reference group, and the effect of the additive genetic relationship between reference group and target group. Target groups were generated by randomly masking a subset of 2,000 animals from the $26,88450 \mathrm{~K}$ animals to either nongenotyped (0K) animals in the scenario of imputing $0 \mathrm{~K}$ animals to $50 \mathrm{~K}$, or to Illumina BovineLD BeadChip (Boichard et al., 2012 ) typed $(\mathbf{7 K})$ animals in the scenario of imputing $7 \mathrm{~K}$ animals to $50 \mathrm{~K}$. In consequence, 24,884 animals remained as the reference group. Not all 0K animals were imputed because FImpute requires that animals in the target group have progenies in the reference group. Smaller reference populations were generated by taking random subsets of sizes 20,000, 15,000, and 5,000 from the complete reference group. To impute the $\mathrm{X}$ chromosome, genotype imputation rules were modified in FImpute to account for the sex chromosome inheritance. For the $\mathrm{X}$ chromosome, the sires of male individuals are set to unknown (Sargolzaei et al., 2014) because males inherit alleles only from the females. To include PAR into imputation, we treated them as autosomal alleles as suggested by Hickey and Kranis (2013). Chromosome 2 (BTA2) was also imputed to compare imputation accuracy with the $\mathrm{X}$ chromosome, because BTA2 is similar in size to the X chromosome.

Imputation accuracies were calculated for SNP on BTA2, X-linked SNP, and SNP in the PAR. Imputation accuracy was measured using 2 statistics: SNP-wise accuracy and animal-wise accuracy. The SNP-wise accuracy was the correlation between the imputed genotype with true genotype both expressed as gene content ( 0 , 1 , or 2). Animal-wise accuracy was calculated for each individual by correlating the true genotypes $(0,1$, or 2$)$ minus the mean gene content per SNP with the imputed genotype $(0,1$, or 2$)$ minus the mean gene content per SNP (Mulder et al., 2012). Mean gene content was calculated as $2 \mathrm{p}$, with $\mathrm{p}$ representing the frequency of the allele for which the homozygote is coded as 2 (Bouwman et al., 2014). For each individual, a cumulative pedigree relationship (CPR) was calculated as the sum of all additive genetic relationships greater than 0.25 with all animals in the reference group. For example, the CPR for animal A with reference group (animal B and $\mathrm{C}$ ) is the sum of the additive genetic relationship between animal $\mathrm{A}$ and $\mathrm{B}$, and animal $\mathrm{A}$ and $\mathrm{C}$. This was done to measure the degree of relationship of this specific animal to the reference group.

The PAR was approximately $5 \mathrm{Mbp}$ long and included 76 SNP, which accounted for $8.5 \%$ of the X chromosome (894) in terms of number of SNP. We compared 
the imputation accuracy when splitting the PAR from the $\mathrm{X}$ chromosome as autosome or not. The results showed a considerable decrease of SNP-wise accuracy of the $\mathrm{X}$ chromosome when the PAR was treated as X linked (Table 1). For example, for a reference group of 24,884 , the SNP-wise accuracy for the X chromosome decreased from 0.94 to 0.92 in the scenario of $0 \mathrm{~K}$ to $50 \mathrm{~K}$ and from 0.93 to 0.90 in the scenario of $7 \mathrm{~K}$ to $50 \mathrm{~K}$. This suggests that the PAR should be imputed separately and treated as autosomal during imputation, which was expected because the PAR is inherited differently from the X-linked region. Software optimized to model the X chromosome and the PAR simultaneously should be developed. However, SNP in the PAR had much lower imputation accuracy compared with X-linked SNP. This is probably because the PAR region is much smaller than the X chromosome and other autosomes. As more phasing errors are expected at the beginning and end of segments, the overall imputation accuracy for the PAR is lower (Sargolzaei et al., 2014). Poor imputation accuracy of SNP in the PAR might also occur if PAR has a higher male recombination rate than X-linked SNP. Human studies have shown that the per-nucleotide recombination rate in the PAR for males is 17 -fold higher than the genome-wide average, due to one obligatory crossing-over per male meiosis in the PAR (Rouyer et al., 1986; Hinch et al., 2014).

The effects of different male/female ratios in the reference group on the imputation accuracy of the $\mathrm{X}$ chromosome and BTA2 are shown in Table 1. In general, more females in the reference group increased the imputation accuracy, especially for the X chromosome.
For SNP-wise accuracy, a reference group of 8,000 males gave an accuracy of 0.76 for the $\mathrm{X}$ chromosome in the scenario of imputing from $0 \mathrm{~K}$ to $50 \mathrm{~K}$, whereas 8,000 females resulted in an accuracy of 0.96 . In addition, more females in the reference group raised the accuracy more when imputing from $0 \mathrm{~K}$ to $50 \mathrm{~K}$ than from $7 \mathrm{~K}$ to $50 \mathrm{~K}$. The reason might be that females provide more information about the $\mathrm{X}$ chromosome than males, because females have 2 copies of the $\mathrm{X}$ chromosome whereas males have only one. This extra information for the $\mathrm{X}$ chromosome could be more beneficial when imputing the nongenotyped individuals. In livestock populations, some elite dams have already been genotyped with high density. As genotyping costs continue to decrease, more females could be genotyped. As a consequence, the $\mathrm{X}$ chromosome could be imputed more accurately in the future using more information from females.

In general, imputation from $0 \mathrm{~K}$ to $50 \mathrm{~K}$ was less accurate than from $7 \mathrm{~K}$ to $50 \mathrm{~K}$. For example, SNP-wise accuracy of $0 \mathrm{~K}$ to $50 \mathrm{~K}$ for BTA2 was on average $10 \%$ lower than $7 \mathrm{~K}$ to $50 \mathrm{~K}$ (Table 1). This result agreed with previous studies (Sargolzaei et al., 2014; Bouwman et al., 2014). If the lower density panel was denser, shared haplotypes between distant relatives can be found more easily and precisely. The results also showed that SNPwise accuracy of $\mathrm{X}$ chromosome is higher than that of BTA2 in the scenario of imputing from $0 \mathrm{~K}$ to $50 \mathrm{~K}$. One explanation might be that males were completely homozygous for the $\mathrm{X}$-linked region of the $\mathrm{X}$ chromosome, so phasing errors for the $\mathrm{X}$ chromosome were lower than for autosomes. Another reason could be due to that imputation of the X-linked and PAR SNP in the

Table 1. The SNP-wise imputation accuracy in different scenarios

\begin{tabular}{|c|c|c|c|c|c|c|c|c|c|}
\hline Item & No. of animals ${ }^{1}$ & Imputed $^{2}$ & \multicolumn{3}{|c|}{$0 \mathrm{~K}-50 \mathrm{~K}^{3}$} & Imputed & \multicolumn{3}{|c|}{$7 \mathrm{~K}-50 \mathrm{~K}^{4}$} \\
\hline $\mathrm{No}_{\mathrm{PAR}}{ }^{7}$ & 24,884 & 899 & 0.89 & 0.92 & $\mathrm{NA}$ & 2,000 & 0.97 & 0.90 & NA \\
\hline & 20,000 & 773 & 0.88 & 0.94 & 0.78 & 2,000 & 0.96 & 0.92 & 0.73 \\
\hline & 15,000 & 642 & 0.87 & 0.94 & 0.77 & 2,000 & 0.96 & 0.90 & 0.63 \\
\hline & 5,000 & 320 & 0.86 & 0.98 & 0.74 & 2,000 & 0.95 & 0.91 & 0.46 \\
\hline \multirow{2}{*}{ Male/female } & $2,000 / 6,000$ & 395 & 0.85 & 0.95 & 0.73 & 2,000 & 0.96 & 0.93 & 0.62 \\
\hline & $0 / 8,000$ & 318 & 0.87 & 0.96 & 0.75 & 2,000 & 0.96 & 0.95 & 0.55 \\
\hline
\end{tabular}

${ }^{1}$ No. of animals $=$ number of animals in the reference group.

${ }^{2}$ Imputed $=$ number of successfully imputed animals.

${ }^{3} 0 \mathrm{~K}-50 \mathrm{~K}=$ scenario of imputing nongenotyped animals to $50 \mathrm{~K}$.

${ }^{4} 7 \mathrm{~K}-50 \mathrm{~K}=$ scenario of imputing $7 \mathrm{~K}$ animals to $50 \mathrm{~K}$.

${ }^{5} \mathrm{X}=\mathrm{X}$ chromosome.

${ }^{6} \mathrm{PAR}=$ pseudoautosomal region.

${ }^{7} \mathrm{No}-\mathrm{PAR}=$ did not treat pseudoautosomal region in the $\mathrm{X}$ chromosome as autosome when imputing. 
scenario of $0 \mathrm{~K}$ to $50 \mathrm{~K}$ had a higher missing rate compared with autosomes, especially when the reference size was small. This might be because $\mathrm{X}$-linked and PAR SNP are quite sparsely genotyped, and shared haplotypes were hard to find with small-size reference groups. In the future, better coverage of the X chromosome should be developed to facilitate genomic studies involving the $\mathrm{X}$ chromosome. Regarding the size of the reference group, imputation accuracy decreased when the size of the reference group was reduced. This observation was in agreement with Sargolzaei et al. (2014).

This study demonstrated that imputation accuracy increased dramatically when the CPR increased, for both $0 \mathrm{~K}$ to $50 \mathrm{~K}$ (Figure 1 ) and $7 \mathrm{~K}$ to $50 \mathrm{~K}$ (results not shown). Most animals had a CPR less than 50. In general, the smaller the CPR, the more variation of animal-wise accuracy. Similarly, the larger the CPR, the higher the animal-wise accuracy. This is probably due to better family information and better population information when CPR is larger. Better family information was reflected by SNP that were imputed more accurately through more progenies, parents, and grandparents. Better population information was reflected by more relatives resulting in more accurate haplotype blocks that the SNP can fit into. These results agree with previous studies (Boison et al., 2014; Bouwman et al., 2014). We also found that imputation from 0K to $50 \mathrm{~K}$ was more affected by CPR. For example, lower CPR often resulted in low imputation accuracy (results were not shown). This is probably because imputation

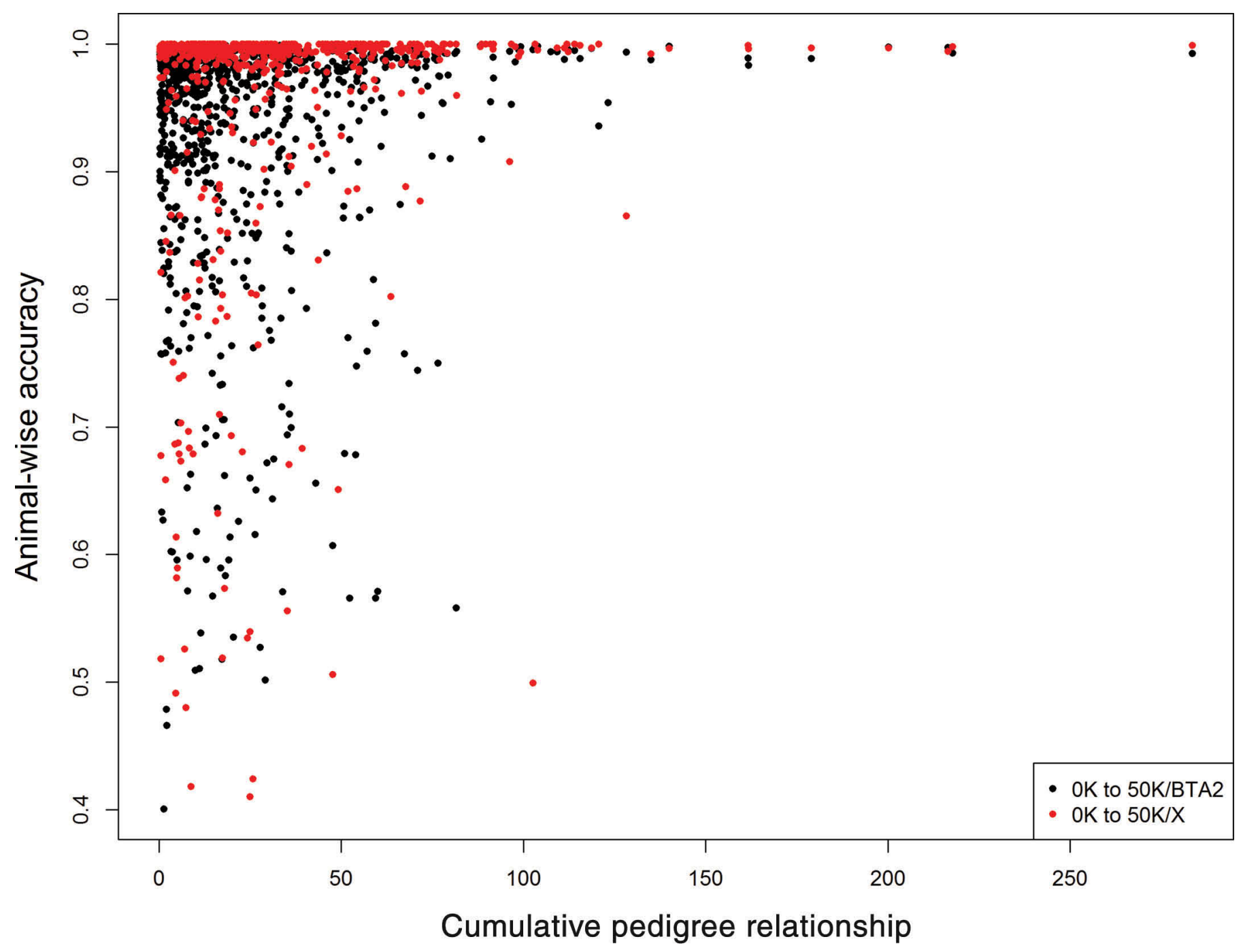

Figure 1. Animal-wise accuracy in the scenario of imputing $0 \mathrm{~K}$ to $50 \mathrm{~K}$, when the size of the reference group is 24,884 . The $\mathrm{x}$-axis is the cumulative pedigree relationship, and the $\mathrm{y}$-axis is the animal-wise accuracy. Black represents the imputation accuracy for chromosome 2 . Red represents the imputation accuracy for the $\mathrm{X}$ chromosome. Color version available online. 
for nongenotyped animals is more dependent on the availability of close family information.

In conclusion, imputation of the $\mathrm{X}$ chromosome was improved if PAR was treated as autosomal because the PAR has inheritance rules that are different from those of the X-linked region. Including more females in the reference group also improved the imputation for the $\mathrm{X}$ chromosome. In general, imputation for nongenotyped animals had lower accuracy compared with animals genotyped with the low-density SNP array. In addition, the larger the CPR between the reference group and the target animal, the higher the imputation accuracy was for this animal. Our study was restricted by limited genotype coverage on the X chromosome, so better coverage of the $\mathrm{X}$ chromosome should be developed to facilitate genomic studies involving the $\mathrm{X}$ chromosome.

\section{ACKNOWLEDGMENTS}

We thank Viking Genetics (Randers, Denmark) for providing blood and semen samples. We acknowledge Solomon Antwi Boison (Nofima, Troms $\varnothing$, Norway) and Peipei Ma (Aarhus University, Tjele, Denmark) for suggestions on the data analyses. XM benefited from an Erasmus-Mundus fellowship within the framework of the European Graduate School in Animal Breeding and Genetics. GS and BG acknowledge the project Genomic Selection-From function to efficient utilization in cattle breeding (grant no. 3412-08-02253), funded jointly by the Danish Ministry of Food, Agriculture and Fisheries (Copenhagen, Denmark), the Milk Levy Fund (Viby, Denmark), Viking Genetics, and Nordic Cattle Genetic Evaluation (Aarhus, Denmark). DJK and AMJ have been financially supported by Mistra Biotech, a research program financed by Mistra-the Swedish foundation for strategic environmental research (Stockholm, Sweden) and the Swedish University of Agricultural Sciences.

\section{REFERENCES}

Boichard, D., H. Chung, R. Dassonneville, X. David, A. Eggen, S. Fritz, K. J. Gietzen, B. J. Hayes, C. T. Lawley, T. S. Sonstegard, C. P. van Tassell, P. M. VanRaden, K. A. Viaud-Martinez, and G. R. Wiggans. 2012. Design of a bovine low-density SNP array optimized for imputation. PLoS ONE 7:e34130. http://dx.doi. org/10.1371/journal.pone.0034130.

Boison, S. A., H. H. R. Neves, A. M. Pérez O'Brien, Y. T. Utsunomiya, R. Carvalheiro, M. V. G. B. da Silva, J. Sölkner, and J. F. Garcia. 2014. Imputation of non-genotyped individuals using genotyped progeny in Nellore, a Bos indicus cattle breed. Livest. Sci. 166:176-189. http://dx.doi.org/10.1016/j.livsci.2014.05.033.

Bouwman, A. C., J. M. Hickey, M. P. Calus, and R. F. Veerkamp. 2014. Imputation of non-genotyped individuals based on genotyped relatives: Assessing the imputation accuracy of a real case scenario in dairy cattle. Genet. Sel. Evol. 46:6. http://dx.doi. org/10.1186/1297-9686-46-6.
Brøndum, R. F., B. Guldbrandtsen, G. Sahana, M. S. Lund, and G. Su. 2014. Strategies for imputation to whole genome sequence using a single or multi-breed reference population in cattle. BMC Genomics 15:728. http://dx.doi.org/10.1186/1471-2164-15-728.

Das, P. J., B. P. Chowdhary, and T. Raudsepp. 2009. Characterization of the bovine pseudoautosomal region and comparison with sheep, goat, and other mammalian pseudoautosomal regions. Cytogenet. Genome Res. 126:139-147. http://dx.doi.org/10.1159/000245913.

de Camargo, G. M. F., L. R. Porto-Neto, M. J. Kelly, R. J. Bunch, S M. McWilliam, H. Tonhati, S. A. Lehnert, M. R. S. Fortes, and S. S. Moore. 2015. Non-synonymous mutations mapped to chromosome $\mathrm{X}$ associated with andrological and growth traits in beef cattle. BMC Genomics 16:384. http://dx.doi.org/10.1186/s12864$015-1595-0$.

Hickey, J. M., and A. Kranis. 2013. Extending long-range phasing and haplotype library imputation methods to impute genotypes on sex chromosomes. Genet. Sel. Evol. 45:10. http://dx.doi. org/10.1186/1297-9686-45-10.

Hinch, A. G., N. Altemose, N. Noor, P. Donnelly, and S. R. Myers. 2014. Recombination in the Human Pseudoautosomal Region PAR1. PLoS Genet. 10:e1004503. http://dx.doi.org/10.1371/ journal.pgen.1004503.

Lyons, R. E., N. T. Loan, L. Dierens, M. R. S. Fortes, M. Kelly, S. S. McWilliam, Y. Li, R. J. Bunch, B. E. Harrison, W. Barendse, S. A. Lehnert, and S. S. Moore. 2014. Evidence for positive selection of taurine genes within a QTL region on chromosome X associated with testicular size in Australian Brahman cattle. BMC Genet. 15:6. http://dx.doi.org/10.1186/1471-2156-15-6.

Ma, P., R. F. Brøndum, Q. Zhang, M. S. Lund, and G. Su. 2013. Comparison of different methods for imputing genome-wide marker genotypes in Swedish and Finnish Red cattle. J. Dairy Sci. 96:4666-4677. http://dx.doi.org/10.3168/jds.2012-6316.

Marchini, J., and B. Howie. 2010. Genotype imputation for genomewide association studies. Nat. Rev. Genet. 11:499-511. http:// dx.doi.org/10.1038/nrg2796.

Matukumalli, L. K., C. T. Lawley, R. D. Schnabel, J. F. Taylor, M. F. Allan, M. P. Heaton, J. O'Connell, S. S. Moore, T. P. L. Smith, T. S. Sonstegard, and C. P. Van Tassell. 2009. Development and characterization of a high density SNP genotyping assay for cattle. PLoS ONE 4:e5350 http://dx.doi.org/10.1371/journal. pone.0005350.

McCarthy, M. I., G. R. Abecasis, L. R. Cardon, D. B. Goldstein, J. Little, J. P. Ioannidis, and J. N. Hirschhorn. 2008. Genomewide association studies for complex traits: Consensus, uncertainty and challenges. Nat. Rev. Genet. 9:356-369. http://dx.doi. org/10.1038/nrg2344.

Meuwissen, T. H. E., B. J. Hayes, and M. E. Goddard. 2001. Prediction of total genetic value using genome-wide dense marker maps. Genetics 157:1819-1829.

Mulder, H. A., M. P. L. Calus, T. Druet, and C. Schrooten. 2012. Imputation of genotypes with low-density chips and its effect on reliability of direct genomic values in Dutch Holstein cattle. J. Dairy Sci. 95:876-889. http://dx.doi.org/10.3168/jds.2011-4490.

Pryce, J. E., J. Johnston, B. J. Hayes, G. Sahana, K. A. Weigel 2nd, S. McParland, D. Spurlock, N. Krattenmacher, R. J. Spelman, E. Wall, and M. P. L. Calus. 2014. Imputation of genotypes from low density (50,000 markers) to high density (700,000 markers) of cows from research herds in Europe, North America, and Australasia using 2 reference populations. J. Dairy Sci. 97:1799-1811. http:// dx.doi.org/10.3168/jds.2013-7368.

Rouyer, F., M. C. Simmler, C. Johnsson, G. Vergnaud, H. J. Cooke, and J. Weissenbach. 1986. A gradient of sex linkage in the pseudoautosomal region of the human sex chromosomes. Nature 319:291295. http://dx.doi.org/10.1038/319291a0.

Sandor, C., F. Farnir, S. Hansoul, W. Coppieters, T. Meuwissen, and M. Georges. 2006. Linkage disequilibrium on the bovine $\mathrm{X}$ chromosome: Characterization and use in quantitative trait locus mapping. Genetics 173:1777-1786. http://dx.doi.org/10.1534/ genetics.106.059329. 
Sargolzaei, M., J. P. Chesnais, and F. S. Schenkel. 2014. A new approach for efficient genotype imputation using information from relatives. BMC Genomics 15:478. http://dx.doi.org/10.1186/14712164-15-478.

Su, G., B. Guldbrandtsen, G. P. Aamand, I. Strandén, and M. S. Lund. 2014. Genomic relationships based on X chromosome markers and accuracy of genomic predictions with and without X chromosome markers. Genet. Sel. Evol. 46:47. http://dx.doi.org/10.1186/12979686-46-47.

van Binsbergen, R., M. C. Bink, M. P. Calus, F. A. van Eeuwijk, B. J. Hayes, I. Hulsegge, and R. F. Veerkamp. 2014. Accuracy of imputation to whole-genome sequence data in Holstein Friesian cattle. Genet. Sel. Evol. 46:41. http://dx.doi.org/10.1186/12979686-46-41.

Van Laere, A. S., W. Coppieters, and M. Georges. 2008. Characterization of the bovine pseudoautosomal boundary: Documenting the evolutionary history of mammalian sex chromosomes. Genome Res. 18:1884-1895. http://dx.doi.org/10.1101/gr.082487.108.

Zimin, A. V., A. L. Delcher, L. Florea, D. R. Kelley, M. C. Schatz, D Puiu, F. Hanrahan, G. Pertea, C. P. Van Tassell, T. S. Sonstegard, G. Marçais, M. Roberts, P. Subramanian, J. A. Yorke, and S. L. Salzberg. 2009. A whole-genome assembly of the domestic cow, Bos taurus. Genome Biol. 10:R42 http://dx.doi.org/10.1186/gb2009-10-4-r42. 